\title{
Application of the Weighted Energy Method in the Partial Fourier Space to Linearized Viscous Conservation Laws with Non-Convex Condition
}

\author{
Yoshihiro Ueda \\ Faculty of Maritime Sciences, Kobe University \\ Japan
}

\section{Introduction}

As you know, the energy method in the Fourier space is useful in deriving the decay estimates for problems in the whole space $\mathbb{R}^{n}$. Recently, the author studied half space problems in $\mathbb{R}_{+}^{n}=$ $\mathbb{R}_{+} \times \mathbb{R}^{n-1}$ and developed the energy method in the partial Fourier space obtained by taking the Fourier transform with respect to the tangential variable $\mathbb{R}^{n-1}$. Then the author applied this energy method to the half space problem for linearized viscous conservation laws with convex condition and proved the asymptotic stability of planar stationary waves by showing a sharp convergence rate for $t \rightarrow \infty$ (see, [14]).

In this chapter, we consider the half space problem for linearized viscous conservation laws with non-convex condition, and derive the asymptotic stability of planar stationary waves and the corresponding convergence rate. Our proof is based on the energy method in the partial Fourier space with the anti-derivative method.

In this present chapter, we are concerned with the half space problem for the viscous conservation laws:

$$
\begin{gathered}
u_{t}-\Delta u+\nabla \cdot f(u)=0, \\
u\left(0, x^{\prime}, t\right)=u_{b}, \\
u(x, 0)=u_{0}(x) .
\end{gathered}
$$

Here $x=\left(x_{1}, \cdots, x_{n}\right)$ is the space variable in the half space $\mathbb{R}_{+}^{n}=\mathbb{R}_{+} \times \mathbb{R}^{n-1}$ with $n \geq 2$; we sometimes write as $x=\left(x_{1}, x^{\prime}\right)$ with $x_{1} \in \mathbb{R}_{+}$and $x^{\prime}=\left(x_{2}, \cdots, x_{n}\right) \in \mathbb{R}^{n-1} ; u(x, t)$ is the unknown function, $u_{0}(x)$ is the initial data satisfying

$$
u_{0}(x) \rightarrow 0 \text { as } \quad x_{1} \rightarrow \infty,
$$

and $u_{b}$ is the boundary data (assumed to be a constant) with $u_{b}<0 ; f(u)=\left(f_{1}(u), \cdots, f_{n}(u)\right)$ is a smooth function of $u \in \mathbb{R}$ with values in $\mathbb{R}^{n}$ and satisfies

$$
f_{1}(0)=0, \quad f_{1}(u)>f_{1}(0)(=0)
$$

for $u \in\left[u_{b}, 0\right)$. Here we note that the condition (1.4) is the necessary condition for the existence of the planar stationary waves (for the detail, see Section 2.2). We emphasize that 
the assumption (1.4) is weaker than the convex condition

$$
f_{1}^{\prime \prime}(u)>0
$$

for $u \in\left[u_{b}, 0\right]$ and $f_{1}(0)=0$. Namely, we do not assume the convex condition for our problem (1.1)-(1.3).

For viscous conservation laws (1.1) with the convex condition (1.5), there are many results on the asymptotic stability of nonlinear waves. First, Il'in and Oleinik in [3] studied the stability of nonlinear waves in the one-dimensional whole space. Liu, Matsumura and Nishihara in the paper [8] discussed the stability of stationary waves in one-dimensional half space. More precisely, they proved the asymptotic stability of several kind of nonlinear waves such as rarefaction waves, stationary waves, and the superposition of stationary waves and rarefaction waves. Later, in a series of papers [5-7], their stability result of stationary waves in one-space dimension was generalized to the multi-dimensional case. Kawashima, Nishibata and Nishikawa [5] first considered the stability of non-degenerate planar stationary waves in two-dimensional half space and obtained the convergence rate $t^{-1 / 4-\alpha / 2}$ in $L^{\infty}$ norm by assuming that the initial perturbation is in $L_{\alpha}^{2}\left(\mathbb{R}_{+} ; L^{2}(\mathbb{R})\right)$. Furthermore, the papers $[6,7]$ studied the $n$-dimensional problem in the $L^{p}$ framework. In particular, the paper [7] showed the stability of non-degenerate planar stationary waves and obtained the convergence rate $t^{-(n / 2)(1 / 2-1 / p)-\alpha / 2}$ in $L^{p}$ norm under the assumption that the initial perturbation belongs to $L_{\alpha}^{2}\left(\mathbb{R}_{+} ; L_{x^{\prime}}^{2}\right)$.

Next, we refer to viscous conservation laws with non-convex condition. Liu and Nishihara in [9] and Nishikawa in [10] investigated the asymptotic stability of travelling waves in the one-dimensional and multi-dimensional whole space, respectively. On the other hand, Hashimoto and Matsumura in [1] studied the asymptotic stability of stationary waves in the one-dimensional half space. Especially, in order to relax the convex condition, Liu and Nishihara in [9] and Nishikawa in [10] employed the anti-derivative method and achieved the desired result. Moreover, Hashimoto and the author in [2] used the same method to derive the asymptotic stability of stationary waves for damped wave equations with non-convex convection term in one-dimensional half space. Inspired by these arguments, we try to relax the convex condition (1.5) and get the asymptotic stability of planar stationary wave for the multi-dimensional problem (1.1)-(1.3). Unfortunately, Nishikawa in the paper [10] considered some special situation for the nonlinear term to make a good combination of the energy method and the anti-derivative method. For the same reason, we will treat the special situation (for the detail, see Section 3).

All these stability results mentioned above are obtained by employing the energy method in the physical space. On the other hand, it is useful to apply the energy method in the partial Fourier space to show sharper convergence rate. Indeed the author's paper [14] considered our problem (1.1)-(1.3) with the convex condition (1.5) and obtained the sharper convergence rate of the planar stationary waves. We shall show the result of the paper [14] in detail.

We are interested in the asymptotic stability of one-dimensional stationary solution $\phi\left(x_{1}\right)$ (called planar stationary wave) for the problem (1.1)-(1.3): $\phi\left(x_{1}\right)$ is a solution to the problem

$$
\begin{gathered}
-\phi_{x_{1} x_{1}}+f_{1}(\phi)_{x_{1}}=0, \\
\phi(0)=u_{b}, \quad \phi\left(x_{1}\right) \rightarrow 0 \quad \text { as } \quad x_{1} \rightarrow \infty .
\end{gathered}
$$


To show the stability, it is convenient to introduce the perturbation $v$ and write the solution $u$ in the form

$$
u(x, t)=\phi\left(x_{1}\right)+v(x, t) .
$$

The original problem (1.1)-(1.3) is then reduced to

$$
\begin{gathered}
v_{t}-\Delta v+\nabla \cdot(f(\phi+v)-f(\phi))=0, \\
v\left(0, x^{\prime}, t\right)=0, \\
v(x, 0)=v_{0}(x),
\end{gathered}
$$

where $v_{0}(x)=u_{0}(x)-\phi\left(x_{1}\right)$; notice that $v_{0}(x) \rightarrow 0$ as $x_{1} \rightarrow \infty$.

Under the convex condition (1.5), the author in [14] showed the asymptotic stability of the planar stationary wave $\phi\left(x_{1}\right)$ by proving a sharp decay estimate for the perturbation $v(x, t)$. To this end we employed the energy method in the partial Fourier space $\hat{\mathbb{R}}_{+}^{n}=\mathbb{R}_{+} \times \mathbb{R}_{\tau}^{n-1}$ which is obtained by taking the Fourier transform with respect to the tangential variable $x^{\prime}=$ $\left(x_{2}, \cdots, x_{n}\right) \in \mathbb{R}^{n-1} ; \xi=\left(\xi_{2}, \cdots, \xi_{n}\right) \in \mathbb{R}_{\xi}^{n-1}$ is the Fourier variable corresponding to $x^{\prime} \in$ $\mathbb{R}^{n-1}$. For the variable $x_{1} \in \mathbb{R}_{+}$in the normal direction, we use $L^{2}$ space (or weighted $L^{2}$ space). As the result, for the corresponding linearized problem with $f(\phi+v)-f(\phi)$ replaced by $f^{\prime}(\phi) v$ in (1.8), we showed the following pointwise estimate with respect to $\xi \in \mathbb{R}_{\xi}^{n-1}$ :

$$
|\mathcal{F} v(\cdot, \xi, t)|_{L^{2}} \leq C e^{-\kappa|\xi|^{2} t}\left|\mathcal{F} v_{0}(\cdot, \xi)\right|_{L^{2}}
$$

where $C$ and $\kappa$ are positive constants. Here $\mathcal{F}$ denotes the Fourier transform with respect to $x^{\prime} \in \mathbb{R}^{n-1}$ and $|\cdot|_{L^{2}}$ is the $L^{2}$ norm with respect to $x_{1} \in \mathbb{R}_{+}$. This pointwise estimate (1.11) enables us to get the following sharp decay estimate:

$$
\|v(t)\|_{\mathcal{L}^{2}} \leq C t^{-(n-1) / 4}\left\|v_{0}\right\|_{L^{2}\left(L^{1}\right)},
$$

where $\|\cdot\|_{\mathcal{L}^{2}}$ denotes the $L^{2}$ norm with respect to $x=\left(x_{1}, x^{\prime}\right) \in \mathbb{R}_{+}^{n},\|\cdot\|_{L^{2}\left(L^{1}\right)}$ is the norm in $L^{2}\left(\mathbb{R}_{+} ; L_{x^{\prime}}^{2} \cap L_{x^{\prime}}^{1}\right)$, and $C$ is a positive constant.

Furthermore, when the planar stationary wave $\phi\left(x_{1}\right)$ is non-degenerate, the author applied the weighted energy method in the partial Fourier space $\hat{\mathbb{R}}_{+}^{n}=\mathbb{R}_{+} \times \mathbb{R}_{\tilde{\xi}}^{n-1}$. Namely, we used the weighted space $L_{\alpha}^{2}$ with respect to $x_{1} \in \mathbb{R}_{+}$. In this case, the pointwise estimate (1.11) is improved to

$$
|\mathcal{F} v(\cdot, \xi, t)|_{L^{2}} \leq C(1+t)^{-\alpha / 2} e^{-\left.\kappa|\xi|\right|^{2} t}\left|\mathcal{F} v_{0}(\cdot, \xi)\right|_{L_{\alpha}^{2}}
$$

where $|\cdot|_{L_{\alpha}^{2}}$ denotes the $L_{\alpha}^{2}$ norm with respect to $x_{1} \in \mathbb{R}_{+}$. Consequently, we had the decay estimate

$$
\|v(t)\|_{\mathcal{L}^{2}} \leq C(1+t)^{-\alpha / 2} t^{-(n-1) / 4}\left\|v_{0}\right\|_{L_{\alpha}^{2}\left(L^{1}\right)},
$$

where $\|\cdot\|_{L_{\alpha}^{2}\left(L^{1}\right)}$ is the norm in $L_{\alpha}^{2}\left(\mathbb{R}_{+} ; L_{x^{\prime}}^{2} \cap L_{x^{\prime}}^{1}\right)$. For the above results, we refer to the reeder [14] in detail.

The main purpose of this chapter is to derive the sharp decay estimate (1.11)-(1.14) for the linearized problem of (1.8)-(1.10) with non-convex condition (1.4), i.e.,

$$
v_{t}-\Delta v+\nabla \cdot\left(f^{\prime}(\phi) v\right)=0
$$


with (1.9), (1.10). To overcome the difficulty occured by the non-convex condition, we make a good combination of the weighted energy method in partial Fourier space employed in [14] and the anti-derivative method employed in $[2,9]$, and get the desired results. Once we obtain the linear stability results for the problem (1.15), (1.9), (1.10), we may apply this results to the asymptotic stability for the nonlinear problem (1.8)-(1.10).

The remainder of this chapter is organized as follows. In Section 2, we introduce function spaces and some preliminaries used in this chapter. Especially, we reformulate our problem (1.15), (1.9), (1.10) by using the anti-derivative method in Section 2.3. In the final section, we treat the half space problem for the reformulated viscous conservation laws and (1.15), (1.9), (1.10), and develop the weighted energy method in the partial Fourier space with the anti-derivative method. In this section, we derive pointwise estimates of solutions and prove the corresponding decay estimates.

\section{Preliminaries}

\subsection{Notations and function spaces}

Let us consider functions defined in the half space $\mathbb{R}_{+}^{n}=\mathbb{R}_{+} \times \mathbb{R}^{n-1}$. We sometimes write the space variable $x=\left(x_{1}, \cdots, x_{n}\right) \in \mathbb{R}_{+}^{n}$ as $x=\left(x_{1}, x^{\prime}\right)$ with $x_{1} \in \mathbb{R}_{+}$and $x^{\prime}=\left(x_{2}, \cdots, x_{n}\right) \in$ $\mathbb{R}^{n-1}$. The symbols $\nabla=\left(\partial_{x_{1}}, \cdots, \partial_{x_{n}}\right)$ and $\Delta=\sum_{j=1}^{n} \partial_{x_{j}}^{2}$ denote the standard gradient and Laplacian with respect to $x=\left(x_{1}, \ldots, x_{n}\right)$, respectively. The symbol $\nabla_{x^{\prime}}=\left(\partial_{x_{2}}, \cdots, \partial_{x_{n}}\right)$ denotes the gradient of the tangential direction with respect to $x=\left(x_{2}, \ldots, x_{n}\right)$. Thus we have $\nabla \cdot g=\sum_{j=1}^{n} \partial_{x_{j}} g_{j}$ for $g=\left(g_{1}, \cdots, g_{n}\right)$, and $\nabla_{x^{\prime}} \cdot g_{*}=\sum_{j=2}^{n} \partial_{x_{j}} g_{j}$ for $g_{*}=\left(g_{2}, \cdots, g_{n}\right)$. Let $\hat{v}\left(x_{1}, \xi\right)$ be the Fourier transform of $v\left(x_{1}, x^{\prime}\right)$ with respect to $x^{\prime} \in \mathbb{R}^{n-1}$ :

$$
\hat{v}\left(x_{1}, \xi\right)=\mathcal{F}\left[v\left(x_{1}, \cdot\right)\right](\xi)=(2 \pi)^{-(n-1) / 2} \int_{\mathbb{R}^{n-1}} v\left(x_{1}, x^{\prime}\right) e^{-i x^{\prime} \cdot \xi} d x^{\prime},
$$

where $\xi=\left(\xi_{2}, \cdots, \xi_{n}\right) \in \mathbb{R}_{\xi}^{n-1}$ is the Fourier variable corresponding to $x^{\prime}=\left(x_{2}, \cdots, x_{n}\right) \in$ $\mathbb{R}^{n-1}$ and $x^{\prime} \cdot \xi=\sum_{j=2}^{n} x_{j} \xi_{j}$.

Let $1 \leq p \leq \infty$. We denote by $L_{x^{\prime}}^{p}=L^{p}\left(\mathbb{R}^{n-1}\right)$ the $L^{p}$ space with respect to $x^{\prime} \in \mathbb{R}^{n-1}$, with the norm $\|\cdot\|_{L_{x^{\prime}}^{p}}$. For a nonnegative integer $s$, we denote by $H_{x^{\prime}}^{s}=H^{s}\left(\mathbb{R}^{n-1}\right)$ the Sobolev space over $\mathbb{R}^{n-1}$ with the norm

$$
\|v\|_{H_{x^{\prime}}^{s}}=\left(\sum_{k=0}^{s}\left\|\partial_{x^{\prime}}^{k} v\right\|_{L_{x^{\prime}}^{2}}^{2}\right)^{1 / 2},
$$

where $\partial_{x^{\prime}}^{k}$ denotes the totality of all the $k$-th order derivatives with respect to $x^{\prime} \in \mathbb{R}^{n-1}$. Also, we denote by $L^{p}\left(\mathbb{R}_{+}\right)$the $L^{p}$ space with respect to $x_{1} \in \mathbb{R}_{+}$, with the norm $|\cdot|_{L^{p}}$. For a nonnegative integer $s$, we denote by $H^{s}\left(\mathbb{R}_{+}\right)$the Sobolev space over $\mathbb{R}_{+}$, with the norm $|\cdot|_{H^{s}}$. For $\alpha \in \mathbb{R}$, we denote by $L_{\alpha}^{2}\left(\mathbb{R}_{+}\right)$the weighted $L^{2}$ space over $\mathbb{R}_{+}$with the norm

$$
|v|_{L_{\alpha}^{2}}=\left(\int_{0}^{\infty}\left(1+x_{1}\right)^{\alpha}\left|v\left(x_{1}\right)\right|^{2} d x_{1}\right)^{1 / 2} .
$$

Now we introduce function spaces over the half space $\mathbb{R}_{+}^{n}=\mathbb{R}_{+} \times \mathbb{R}^{n-1}$. Let $1 \leq p, q \leq \infty, s$ be a nonnegative integer, and $\alpha \in \mathbb{R}$. The space $L^{q}\left(L^{p}\right)=L^{q}\left(\mathbb{R}_{+} ; L_{x^{\prime}}^{p}\right)$ consists of $L^{q}$ functions 
of $x_{1} \in \mathbb{R}_{+}$with values in $L_{x^{\prime}}^{p}$ with respect to $x^{\prime} \in \mathbb{R}^{n-1}$. The norm is denoted by $\|\cdot\|_{L^{q}\left(L^{p}\right)}$. When $q=p$, we simply write as

$$
\mathcal{L}^{p}=L^{p}\left(L^{p}\right), \quad\|\cdot\|_{\mathcal{L}^{p}}=\|\cdot\|_{L^{p}\left(L^{p}\right)} \cdot
$$

The space $H^{s}\left(L^{p}\right)=H^{s}\left(\mathbb{R}_{+} ; L_{x^{\prime}}^{p}\right)$ consists of $H^{s}$ functions of $x_{1} \in \mathbb{R}_{+}$with values in $L_{x^{\prime}}^{p}$ with respect to $x^{\prime} \in \mathbb{R}^{n-1}$. The norm is denoted by $\|\cdot\|_{H^{s}\left(L^{p}\right)}$. Also, $L_{\alpha}^{2}\left(L^{p}\right)=L_{\alpha}^{2}\left(\mathbb{R}_{+} ; L_{x^{\prime}}^{p}\right)$ denotes the space of $L_{\alpha}^{2}$ functions of $x_{1} \in \mathbb{R}_{+}$with values in $L_{x^{\prime}}^{p}$ with respect to $x^{\prime} \in \mathbb{R}^{n-1}$. The norm is denoted by

$$
\|v\|_{L_{\alpha}^{2}\left(L^{p}\right)}=\left(\int_{0}^{\infty}\left(1+x_{1}\right)^{\alpha}\left\|v\left(x_{1}, \cdot\right)\right\|_{L_{x^{\prime}}^{p}}^{2} d x_{1}\right)^{1 / 2} .
$$

We sometimes use

$$
\mathcal{L}_{\alpha}^{2}=L_{\alpha}^{2}\left(L^{2}\right), \quad\|\cdot\|_{\mathcal{L}_{\alpha}^{2}}=\|\cdot\|_{L_{\alpha}^{2}\left(L^{2}\right)} .
$$

$L^{2}\left(H^{s}\right)=L^{2}\left(\mathbb{R}_{+} ; H_{x^{\prime}}^{s}\right)$ denotes the space of $L^{2}$ functions of $x_{1} \in \mathbb{R}_{+}$with values in $H_{x^{\prime}}^{s}$ with respect to $x^{\prime} \in \mathbb{R}^{n-1}$, whose norm is given by

$$
\begin{aligned}
\|v\|_{L^{2}\left(H^{s}\right)} & =\left(\int_{0}^{\infty}\left\|v\left(x_{1}, \cdot\right)\right\|_{H_{x^{\prime}}^{s}}^{2} d x_{1}\right)^{1 / 2} \\
& =\left(\sum_{k=0}^{s} \int_{0}^{\infty}\left\|\partial_{x^{\prime}}^{k} v\left(x_{1}, \cdot\right)\right\|_{L_{x^{\prime}}^{2}}^{2} d x_{1}\right)^{1 / 2}=\left(\sum_{k=0}^{s}\left\|\partial_{x^{\prime}}^{k} v\right\|_{\mathcal{L}^{2}}^{2}\right)^{1 / 2} .
\end{aligned}
$$

By the definition (2.1) of the Fourier transform, we see that

$$
\sup _{\xi \in \mathbb{R}_{\tilde{\zeta}}^{n-1}}|\hat{v}(\cdot, \xi)|_{L^{2}} \leq C\|v\|_{L^{2}\left(L^{1}\right)}
$$

with $C=(2 \pi)^{-(n-1) / 2}$. Also, it follows from the Plancherel theorem that

$$
\left\|\partial_{x^{\prime}}^{k} v\right\|_{\mathcal{L}_{\alpha}^{2}}=\left(\int_{\mathbb{R}_{\xi}^{n-1}}|\xi|^{2 k}|\hat{v}(\cdot, \xi)|_{L_{\alpha}^{2}}^{2} d \xi\right)^{1 / 2}
$$

Let $T>0$ and let $X$ be a Banach space defined on the half space $\mathbb{R}_{+}^{n}$. Then $C([0, T] ; X)$ denotes the space of continuous functions of $t \in[0, T]$ with values in $X$.

In this paper, positive constants will be denoted by $C$ or $c$.

\subsection{Stationary solution}

We review the results on the stationary problem (1.6)-(1.7). For the details, we refer the reader to $[2,8,11-13]$.

Proposition 2.1 ([8]). Assume the condition (1.4). Then $f_{1}^{\prime}(0) \leq 0$ is necessary for the existence of solutions to the stationary problem (1.6)-(1.7). Conversely, under the condition $f_{1}^{\prime}(0) \leq 0$, we have the following existence result:

(i) Non-degenerate case where $f_{1}^{\prime}(0)<0$ : In this case the stationary problem (1.6)-(1.7) admits a unique smooth solution $\phi\left(x_{1}\right)$ with $\phi_{x_{1}}>0$ (resp. $\phi_{x_{1}}<0$ ), provided that $u_{b}<0$ (resp. $0<u_{b}$ and $f_{1}(u)<f_{1}(0)$ for $\left.0<u<u_{b}\right)$. The solution verifies

$$
\left|\phi\left(x_{1}\right)\right| \leq C e^{-c x_{1}}, \quad x_{1}>0,
$$


where $C$ and $c$ are positive constants.

(ii) Degenerate case where $f_{1}^{\prime}(0)=0$ : In this case the problem (1.6)-(1.7) admits a unique smooth solution $\phi\left(x_{1}\right)$ if and only if $u_{b}<0$. The solution verifies $\phi_{x_{1}}>0$ and

$$
\left|\phi\left(x_{1}\right)\right| \leq C\left(1+x_{1}\right)^{-1 / q}, \quad x_{1}>0,
$$

where $q$ is the degeneracy exponent of $f_{1}$ and $C$ is a positive constant.

In this chapter we only treat the stationary solutions $\phi\left(x_{1}\right)$ with $\phi_{x_{1}}>0$ and discuss their stability; however, we must get the similar stability result of the monotone decreasing stationary solutions by using the same argument introduced in this chapter. (We refer the reader to [2].)

\subsection{Reformulated problem}

In this subsection we reformulate our problem by the anti-derivative method. To this end we introduce a new function $z(x, t)$ as

$$
z(x, t)=-\int_{x_{1}}^{\infty} v\left(y, x^{\prime}, t\right) d y .
$$

Here, we assume the integrability of $v(x, t)$ over $\mathbb{R}_{+}$. This transformation is motivated by the argument in Liu-Nishihara [9]. By using (2.4), we can reformulate (1.8)-(1.10) in terms of $z(x, t)$ as

$$
\begin{gathered}
z_{t}-\Delta z+f^{\prime}(\phi) \cdot \nabla z+\int_{x_{1}}^{\infty} \phi_{x_{1}} f_{*}^{\prime \prime}(\phi) \cdot \nabla_{x^{\prime}} z d y=-g_{1}+\nabla_{x^{\prime}} \cdot h_{* \prime} \\
z_{x_{1}}\left(0, x^{\prime}, t\right)=0, \\
z(x, 0)=z_{0}(x),
\end{gathered}
$$

where $z_{0}(x)=-\int_{x_{1}}^{\infty}\left(u_{0}\left(y, x^{\prime}\right)-\phi(y)\right) d y, f_{*}^{\prime \prime}(\phi)=\left(f_{2}^{\prime \prime}(\phi), \cdots, f_{n}^{\prime \prime}(\phi)\right)$, and $g_{1}, \nabla_{x^{\prime}} \cdot h_{*}$ are nonlinear terms defined by $h_{*}=\left(h_{2}, \cdots, h_{n}\right)$ and

$$
g_{j}=f_{j}\left(\phi+z_{x_{1}}\right)-f_{j}(\phi)-f_{j}^{\prime}(\phi) z_{x_{1}}, \quad h_{j}=\int_{x_{1}}^{\infty} g_{j} d y .
$$

Once we obtain the solution for the problem (2.5)-(2.7), the differentiation $v=z_{x_{1}}$ is the solution for (1.8)-(1.10). Namely, we will apply the weighted energy method in the partial Fourier space and try ot derive the global solution in time to the reformulated problem (2.5)-(2.7). We will discuss this reformulated problem in Section 3 to prove our main theorems.

\subsection{Weight function}

We introduce the weight function employed in the weighted energy method. Our weight function is defined as

$$
w(u)=\left(-e^{A u}+1\right) / f_{1}(u) \quad \text { for } \quad u \in\left[u_{b}, 0\right],
$$


where $A$ is a positive constant determined in Lemma 2.2. This weight function is very important to derive a priori estimate in the latter section. For this weight function, we obtain the following lemma.

Lemma 2.2 ([2]). Suppose that $f_{1}(u)$ satisfies (1.4). Let $w(u)$ be the weight function defined in (2.8). Then there exists a positive constant $\delta$ such that if $A \geq \delta$, then $w(u)$ satisfies the following conditions:

$$
\begin{array}{ll}
\text { (i) }\left(w f_{1}\right)^{\prime}(u)<0 & \text { for } \quad u \in\left[u_{b}, 0\right], \\
\text { (ii) }\left(w f_{1}\right)^{\prime \prime}(u)<0 & \text { for } \quad u \in\left[u_{b}, 0\right] .
\end{array}
$$

Moreover, let $\phi$ be the stationary solution constructed in Proposition 2.1. Then the weight function satisfies the following properties.

(i) Non-degenerate case where $f_{1}^{\prime}(0)<0$ : The weight function $w(\phi)$ satisfies

$$
c<w(\phi)<C \quad \text { for } \quad \phi \in\left[u_{b}, 0\right] .
$$

(ii) Degenerate case where $f_{1}^{\prime}(0)=0$ : The weight function $w(\phi)$ satisfies

$$
c\left(1+x_{1}\right)<w(\phi)<C\left(1+x_{1}\right) \quad \text { for } \quad \phi \in\left[u_{b}, 0\right] .
$$

Here, $C$ and $c$ are some positive constants which independent of $x_{1}$.

The detail of the proof is omitted here. For the details, we refer the reader to [2].

\section{Asymptotic stability with convergence rates}

In the final section, we apply our weighted energy method in the partial Fourier space to the linearized problem. We consider the linearized problem corresponding to the half space problem (2.5)-(2.7). Namely, we consider (2.5) with $g_{j}=0$ for $j=1, \cdots, n$. For this linearized equation, we treat the special situation that

$$
\text { " } f_{j}(u) \text { are linear in } u \in\left[u_{b}, 0\right] \text { for } j=2, \cdots, n . "
$$

Then our initial value problem of the linearized equation is written as

$$
z_{t}-\Delta z+f^{\prime}(\phi) \cdot \nabla z=0
$$

together with (2.6) and (2.7). Taking the Fourier transform with respect to $x^{\prime} \in \mathbb{R}^{n-1}$ for the linearized problem (3.1), (2.6), (2.7), we obtain

$$
\begin{aligned}
& \hat{z}_{t}-\hat{z}_{x_{1} x_{1}}+|\xi|^{2} \hat{z}+f_{1}^{\prime}(\phi) \hat{z}_{x_{1}}+i \xi \cdot f_{*}^{\prime}(\phi) \hat{z}=0, \\
& \hat{z}_{x_{1}}(0, \xi, t)=0, \\
& \hat{z}\left(x_{1}, \xi, 0\right)=\hat{z}_{0}\left(x_{1}, \xi\right),
\end{aligned}
$$

where $\xi=\left(\xi_{2}, \cdots, \xi_{n}\right) \in \mathbb{R}_{\xi}^{n-1}$ is the Fourier variable corresponding to $x^{\prime}=\left(x_{2}, \cdots, x_{n}\right) \in$ $\mathbb{R}^{n-1}, f_{*}^{\prime}(\phi)=\left(f_{2}^{\prime}(\phi), \cdots, f_{n}^{\prime}(\phi)\right)$, and $\xi \cdot f_{*}^{\prime}(\phi)=\sum_{j=2}^{n} \xi_{j} f_{j}^{\prime}(\phi)$. This is the formulation of our linearized problem in the partial Fourier space $\hat{\mathbb{R}}_{+}^{n}=\mathbb{R}_{+} \times \mathbb{R}_{\xi}^{n-1}$. 
Furthermore, we sometimes use the differentiated problem. We differentiate the problem (3.1), (2.6), (2.7) with respect to $x_{1}$. Then this yields our problem (1.15) together with (1.9) and (1.10), and the corresponding problem in the partial Fourier space:

$$
\begin{aligned}
& \hat{v}_{t}-\hat{v}_{x_{1} x_{1}}+|\xi|^{2} \hat{v}+\left(f_{1}^{\prime}(\phi) \hat{v}\right)_{x_{1}}+i \xi \cdot f_{*}^{\prime}(\phi) \hat{v}=0, \\
& \hat{v}(0, \xi, t)=0, \\
& \hat{v}\left(x_{1}, \xi, 0\right)=\hat{v}_{0}\left(x_{1}, \xi\right) .
\end{aligned}
$$

Here we note that $v=z_{x_{1}}$. By applying the weighted energy method to the above problems, we obtain the pointwise estimate of solutions.

\subsection{Energy method}

We apply the energy method to the problems (3.2) and (3.3) formulated in the partial Fourier space and derive pointwise estimates of solutions to (3.2). We use $L^{2}$ space for the variable $x_{1} \in \mathbb{R}_{+}$in the normal direction. The result is given as follows.

Theorem 3.1 (Pointwise estimate). Let $\phi\left(x_{1}\right)$ be a stationary solution with $\phi_{x_{1}}>0$. Then the solution to the problem (3.2) verifies the following pointwise estimate.

(i) Non-degenerate case where $f_{1}^{\prime}(0)<0$ : Suppose that $\hat{z}_{0}(\cdot, \xi) \in H^{2}\left(\mathbb{R}_{+}\right)$for each $\xi \in \mathbb{R}_{\xi}^{n-1}$. Then it holds

$$
\begin{aligned}
|\hat{z}(\cdot, \xi, t)|_{L_{\alpha}^{2}} & \leq C e^{-\kappa|\xi|^{2} t}\left|\hat{z}_{0}(\cdot, \xi)\right|_{L_{\alpha^{\prime}}^{2}} \\
\left|\hat{z}_{x_{1}}(\cdot, \xi, t)\right|_{L^{2}} & \leq C e^{-\kappa|\xi|^{2} t}\left(\left|\hat{z}_{0}(\cdot, \xi)\right|_{L_{\alpha}^{2}}+\left|\left(\hat{z}_{0}\right)_{x_{1}}(\cdot, \xi)\right|_{L^{2}}\right), \\
\left|\hat{z}_{x_{1} x_{1}}(\cdot, \xi, t)\right|_{L^{2}} & \leq C e^{-\left.\kappa|\xi|\right|^{2} t}\left(\left|\hat{z}_{0}(\cdot, \xi)\right|_{L_{\alpha}^{2}}+\left|\left(\hat{z}_{0}\right)_{x_{1}}(\cdot, \xi)\right|_{H^{1}}\right)
\end{aligned}
$$

with $\alpha=0$, for $\xi \in \mathbb{R}_{\xi}^{n-1}$ and $t \geq 0$, where $|\cdot|_{L_{\alpha}^{2}}$ denotes the $L_{\alpha}^{2}$ norm with respect to $x_{1} \in \mathbb{R}_{+}$, and $C$ and $\kappa$ are positive constants.

(ii) Degenerate case where $f_{1}^{\prime}(0)=0$ : Suppose that $\hat{z}_{0}(\cdot, \xi) \in L_{1}^{2}\left(\mathbb{R}_{+}\right)$and $\left(\hat{z}_{0}\right)_{x_{1}}(\cdot, \xi) \in H^{1}\left(\mathbb{R}_{+}\right)$ for each $\xi \in \mathbb{R}_{\xi}^{n-1}$. Then it holds that (3.4)-(3.6) with $\alpha=1$.

As a simple corollary we have the following decay estimate.

Corollary 3.2 (Decay estimate). Assume the same conditions of Proposition 3.1. Then the solution to the problem (3.1), (2.6), (2.7) satisfies the following decay estimate.

(i) Non-degenerate case where $f_{1}^{\prime}(0)<0$ : Suppose that $z_{0} \in H^{2}\left(L^{1}\right)$. Then this yields

$$
\begin{aligned}
& \left\|\partial_{x^{\prime}}^{k} z(t)\right\|_{\mathcal{L}_{\alpha}^{2}} \leq C t^{-(n-1) / 4-k / 2}\left\|z_{0}\right\|_{L_{\alpha}^{2}\left(L^{1}\right)}, \\
& \left\|\partial_{x^{\prime}}^{k} z_{x_{1}}(t)\right\|_{\mathcal{L}^{2}} \leq C t^{-(n-1) / 4-k / 2}\left(\left\|z_{0}\right\|_{L_{\alpha}^{2}\left(L^{1}\right)}+\left\|\left(z_{0}\right)_{x_{1}}\right\|_{L^{2}\left(L^{1}\right)}\right), \\
& \left\|\partial_{x^{\prime}}^{k} z_{x_{1} x_{1}}(t)\right\|_{\mathcal{L}^{2}} \leq C t^{-(n-1) / 4-k / 2}\left(\left\|z_{0}\right\|_{L_{\alpha}^{2}\left(L^{1}\right)}+\left\|\left(z_{0}\right)_{x_{1}}\right\|_{H^{1}\left(L^{1}\right)}\right)
\end{aligned}
$$

with $\alpha=0$, for $t>0$, where $k \geq 0$ is an integer and $C$ is a positive constant. 
(ii) Degenerate case where $f_{1}^{\prime}(0)=0$ : Suppose that $z_{0} \in L_{1}^{2}\left(L^{1}\right)$ and $\left(z_{0}\right)_{x_{1}} \in H^{1}\left(L^{1}\right)$. Then this yields that (3.7)-(3.9) with $\alpha=1$.

Proof of Theorem 3.1. Throughout this proof, we use the weighted $L^{2}$ norm:

$$
|a|_{L_{w}^{2}}=\left(\int_{0}^{\infty} w\left(\phi\left(x_{1}\right)\right)\left|a\left(x_{1}\right)\right|^{2} d x_{1}\right)^{1 / 2},
$$

where $w$ is the weight function defined by (2.8). For this weighted norm, by using Lemma 2.2, we see the following properties.

$$
\begin{aligned}
& c|\cdot|_{L^{2}} \leq|\cdot|_{L_{w}^{2}} \leq C|\cdot|_{L^{2}} \text { for the non-degenerate case : } f^{\prime}(0)<0, \\
& c|\cdot|_{L_{1}^{2}} \leq|\cdot|_{L_{w}^{2}} \leq C|\cdot|_{L_{1}^{2}} \text { for the degenerate case : } f^{\prime}(0)=0 .
\end{aligned}
$$

We prove (i) and (ii) in Theorem 3.1 in parallel. We first derive (3.4). We multiply (3.2) $)_{1}$ by $w(\phi) \bar{z}$ and take the real part, obtaining

$$
\frac{1}{2} w(\phi) \frac{\partial}{\partial t}|\hat{z}|^{2}+\frac{\partial}{\partial x_{1}} \mathcal{F}_{1}+\mathcal{D}_{1}=0
$$

where

$$
\begin{aligned}
& \mathcal{D}_{1}=w(\phi)\left(\left|\hat{z}_{x_{1}}\right|^{2}+\left.|\xi|\right|^{2}|\hat{z}|^{2}\right)-\frac{1}{2}\left(w f_{1}\right)^{\prime \prime}(\phi) \phi_{x_{1}}|\hat{z}|^{2}, \\
& \mathcal{F}_{1}=\frac{1}{2}\left(w f_{1}\right)^{\prime}(\phi)|\hat{z}|^{2}-w(\phi) \operatorname{Re}\left(\overline{\hat{z}} \hat{z}_{x_{1}}\right),
\end{aligned}
$$

and $w$ is a weight function defined by (2.8). By virtue of $(2.9)_{2}$ in Lemma 2.2, we have

$$
\mathcal{D}_{1} \geq c w(\phi)\left(\left|\hat{z}_{x_{1}}\right|^{2}+|\xi|^{2}|\hat{z}|^{2}\right)+c \phi_{x_{1}}|\hat{z}|^{2},
$$

where $c$ is a some positive constant. Therefore, integrating (3.11) in $x_{1} \in \mathbb{R}_{+}$, we get

$$
\frac{\partial}{\partial t}|\hat{z}|_{L_{w}^{2}}^{2}+c_{1} \hat{D}_{1}-\left(w f_{1}\right)^{\prime}\left(u_{b}\right)|\hat{z}(0, \xi, t)|^{2} \leq 0
$$

with a positive constant $c_{1}$, where

$$
\hat{D}_{1}=\left|\hat{z}_{x_{1}}\right|_{L_{w}^{2}}^{2}+|\xi|^{2}|\hat{z}|_{L_{w}^{2}}^{2}+\left|\sqrt{\phi_{x_{1}}} \hat{z}\right|_{L^{2}}^{2} .
$$

Here, by virtue of $(2.9)_{1}$, the last term of the left-hand side of (3.13) is positive. We multiply (3.13) by $\left.e^{\kappa \mid \xi}\right|^{2} t(\kappa>0)$ to get

$$
\frac{\partial}{\partial t}\left(\left.e^{\kappa \mid \xi}\right|^{2} t|\hat{z}|_{L_{w}^{2}}^{2}\right)+e^{\kappa|\xi|^{2} t}\left(c_{1} \hat{D}_{1}-\kappa|\xi|^{2}|\hat{z}|_{L_{w}^{2}}^{2}\right) \leq 0 .
$$

Noting that $\hat{D}_{1} \geq|\xi|^{2}|\hat{z}|_{L_{w}^{2}}^{2}$, we choose $\kappa>0$ such that $\kappa<c_{1}$ and integrate (3.15) over $[0, t]$. This yields

$$
e^{\kappa|\xi|^{2} t}|\hat{z}(\cdot, \xi, t)|_{L_{w}^{2}}^{2}+\int_{0}^{t} e^{\kappa|\xi|^{2} \tau} \hat{D}_{1}(\xi, \tau) d \tau \leq C\left|\hat{z}_{0}(\cdot, \xi)\right|_{L_{w}^{2}}^{2}
$$


where $C$ is a positive constant.

We next prove (3.5). Multiplying (3.3) ${ }_{1}$ by $\overline{\hat{v}}$ and taking the real part, then we have

$$
\frac{1}{2} \frac{\partial}{\partial t}|\hat{v}|^{2}+\frac{\partial}{\partial x_{1}} \mathcal{F}_{2}+\mathcal{D}_{2}=0
$$

where

$$
\begin{aligned}
& \mathcal{D}_{2}=\left|\hat{v}_{x_{1}}\right|^{2}+|\xi|^{2}|\hat{v}|^{2}+\frac{1}{2} f_{1}^{\prime \prime}(\phi) \phi_{x_{1}}|\hat{v}|^{2}, \\
& \mathcal{F}_{2}=\frac{1}{2} f_{1}^{\prime}(\phi)|\hat{v}|^{2}-\operatorname{Re}\left(\overline{\hat{v}}_{x_{x_{1}}}\right) .
\end{aligned}
$$

We integrate (3.17) in $x_{1} \in \mathbb{R}_{+}$to obtain

$$
\frac{\partial}{\partial t}|\hat{v}|_{L^{2}}^{2}+2 \hat{D}_{2} \leq C|\hat{v}|_{L^{2}}^{2}
$$

where $\hat{D}_{2}=\left|\hat{v}_{x_{1}}\right|_{L^{2}}^{2}+|\xi|^{2}|\hat{v}|_{L^{2}}^{2}$ and $C$ is a positive constant. We multiply (3.18) by $\left.e^{\kappa \mid \xi}\right|^{2} t(\kappa>0)$ to get

$$
\frac{\partial}{\partial t}\left(e^{\left.\kappa|\xi|\right|^{2} t}|\hat{v}|_{L^{2}}^{2}\right)+e^{\kappa|\xi|^{2} t}\left(2 \hat{D}_{2}-\kappa|\xi|^{2}|\hat{v}|_{L^{2}}^{2}\right) \leq C e^{\kappa|\xi|^{2} t}|\hat{v}|_{L^{2}}^{2}
$$

Then we choose $\kappa>0$ such that $\kappa<2$ and integrate (3.19) over $[0, t]$. This yields

$$
\left.e^{\kappa \mid \xi}\right|^{2} t|\hat{v}(\cdot, \xi, t)|_{L^{2}}^{2}+\left.\int_{0}^{t} e^{\kappa \mid \xi}\right|^{2} \tau \hat{D}_{2}(\xi, \tau) d \tau \leq C\left|\hat{v}_{0}(\cdot, \xi)\right|_{L^{2}}^{2}+\left.C \int_{0}^{t} e^{\kappa \mid \xi}\right|^{2} \tau|\hat{v}(\cdot, \xi, \tau)|_{L^{2}}^{2} d \tau .
$$

Noting that $v=z_{x_{1}}$ and $|\hat{v}|_{L^{2}} \leq C|\hat{v}|_{L_{w}^{2}}$, we apply (3.16) to the above inequality. Then we get

$$
\left.e^{\kappa \mid \xi}\right|^{2} t|\hat{v}(\cdot, \xi, t)|_{L^{2}}^{2}+\left.\int_{0}^{t} e^{\kappa \mid \xi}\right|^{2} \tau \hat{D}_{2}(\xi, \tau) d \tau \leq C\left(\left|\hat{z}_{0}(\cdot, \xi)\right|_{L_{w}^{2}}^{2}+\left|\hat{v}_{0}(\cdot, \xi)\right|_{L^{2}}^{2}\right) .
$$

We shall show (3.6). Multiplying (3.3) 1 by $-\overline{\hat{v}}_{x_{1} x_{1}}$ and taking the real part, then we have

$$
\frac{1}{2} \frac{\partial}{\partial t}\left|\hat{v}_{x_{1}}\right|^{2}+\frac{\partial}{\partial x_{1}} \mathcal{F}_{3}+\mathcal{D}_{3}=0
$$

where

$$
\begin{gathered}
\mathcal{D}_{3}=\left|\hat{v}_{x_{1} x_{1}}\right|^{2}+|\xi|^{2}\left|\hat{v}_{x_{1}}\right|^{2}+\frac{3}{2} f_{1}^{\prime \prime}(\phi) \phi_{x_{1}}\left|\hat{v}_{x_{1}}\right|^{2} \\
-\frac{1}{2}\left(\left(f_{1} f_{1}^{\prime \prime}\right)^{\prime} f_{1}\right)^{\prime}(\phi) \phi_{x_{1}}|\hat{v}|^{2}-\frac{1}{2} i \xi\left(f_{1} f_{*}^{\prime \prime}\right)^{\prime}(\phi) \phi_{x_{1}}|\hat{v}|^{2}, \\
\mathcal{F}_{3}=\frac{1}{2} f_{1}^{\prime}(\phi)\left|\hat{v}_{x_{1}}\right|^{2}+\frac{1}{2}\left\{\left(f_{1} f_{1}^{\prime \prime}\right)^{\prime}(\phi)+i \xi f_{*}^{\prime \prime}(\phi)\right\} \phi_{x_{1}}|\hat{v}|^{2} \\
-\operatorname{Re}\left(\hat{v}_{t} \overline{\hat{v}}_{x_{1}}\right)-\left(|\xi|^{2}+f_{1}^{\prime \prime}(\phi) \phi_{x_{1}}+i \xi f_{*}^{\prime}(\phi)\right) \operatorname{Re}\left(\hat{v} \overline{\hat{v}}_{x_{1}}\right) .
\end{gathered}
$$


Integrating (3.21) in $x_{1} \in \mathbb{R}_{+}$, we have

$$
\frac{\partial}{\partial t}\left|\hat{v}_{x_{1}}\right|_{L^{2}}^{2}+2 \hat{D}_{3} \leq C\left(|\hat{v}|_{H^{1}}^{2}+|\xi||\hat{v}|_{L^{2}}^{2}\right),
$$

where $\hat{D}_{3}=\left|\hat{v}_{x_{1} x_{1}}\right|_{L^{2}}^{2}+|\xi|^{2}\left|\hat{v}_{x_{1}}\right|_{L^{2}}^{2}$ and $C$ is a positive constant. We multiply (3.22) by $\left.e^{\kappa \mid \xi}\right|^{2} t$ $(\kappa>0)$ to get

$$
\frac{\partial}{\partial t}\left(e^{\kappa|\xi|^{2} t}\left|\hat{v}_{x_{1}}\right|_{L^{2}}^{2}\right)+e^{\kappa|\xi|^{2} t}\left(2 \hat{D}_{3}-\kappa|\xi|^{2}\left|\hat{v}_{x_{1}}\right|_{L^{2}}^{2}\right) \leq C e^{\kappa|\xi|^{2} t}\left(|\hat{v}|_{H^{1}}^{2}+|\xi||\hat{v}|_{L^{2}}^{2}\right) .
$$

Then we choose $\kappa>0$ such that $\kappa<2$ and integrate (3.23) over $[0, t]$. This yields

$$
\begin{aligned}
& e^{\kappa|\xi|^{2} t}\left|\hat{v}_{x_{1}}(\cdot, \xi, t)\right|_{L^{2}}^{2}+\int_{0}^{t} e^{\kappa|\xi|^{2} \tau} \hat{D}_{3}(\xi, \tau) d \tau \\
& \leq C\left|\left(\hat{v}_{0}\right)_{x_{1}}(\cdot, \xi)\right|_{L^{2}}^{2}+C \int_{0}^{t} e^{\kappa|\xi|^{2} \tau}\left(|\hat{v}(\cdot, \xi, \tau)|_{H^{1}}^{2}+|\xi|^{2}|\hat{v}(\cdot, \xi, \tau)|_{L^{2}}^{2}\right) d \tau .
\end{aligned}
$$

Thus, employing (3.16) and (3.20) to the above inequality, we obtain

$$
e^{\kappa|\xi|^{2} t}\left|\hat{v}_{x_{1}}(\cdot, \xi, t)\right|_{L^{2}}^{2}+\left.\int_{0}^{t} e^{\kappa \mid \xi}\right|^{2} \tau \hat{D}_{3}(\xi, \tau) d \tau \leq C\left(\left|\hat{z}_{0}(\cdot, \xi)\right|_{L_{w}^{2}}^{2}+\left|\hat{v}_{0}(\cdot, \xi)\right|_{H^{1}}^{2}\right) .
$$

Finally, we apply (3.10) to the estimates (3.16), (3.20) and (3.24). Then this gives the desired estimates (3.4)-(3.6) with $\alpha=0,1$. Hence the proof of Theorem 3.1 is completed.

Here, for later use, we derive the corresponding time weighted estimate. We multiply (3.15) (or (3.19), (3.23)) with $0<\kappa \leq c_{1}$ (or $\left.0<\kappa<2\right)$ by $(1+t)^{\gamma}(\gamma \geq 0)$ and integrate over $[0, t]$. Then this yields the desired estimate:

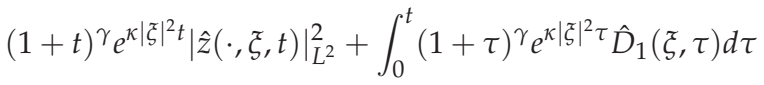

$$
\begin{aligned}
& \leq C\left|\hat{z}_{0}(\cdot, \xi)\right|_{L^{2}}^{2}+\left.\gamma C \int_{0}^{t}(1+\tau)^{\gamma-1} e^{\kappa \mid \xi}\right|^{2} \tau|\hat{z}(\cdot, \xi, \tau)|_{L^{2}}^{2} d \tau, \\
& (1+t)^{\gamma} e^{\kappa|\xi|^{2} t}|\hat{v}(\cdot, \xi, t)|_{L^{2}}^{2}+\int_{0}^{t}(1+\tau)^{\gamma} e^{\kappa|\xi|^{2} \tau} \hat{D}_{2}(\xi, \tau) d \tau \\
& \leq C\left|\hat{v}_{0}(\cdot, \xi)\right|_{L^{2}}^{2}+C \int_{0}^{t}(1+\tau)^{\gamma} e^{\kappa|\xi|^{2} \tau}|\hat{v}(\cdot, \xi, \tau)|_{L^{2}}^{2} d \tau \\
& (1+t)^{\gamma} e^{\kappa|\xi|^{2} t}\left|\hat{v}_{x_{1}}(\cdot, \xi, t)\right|_{L^{2}}^{2}+\int_{0}^{t}(1+\tau)^{\gamma} e^{\kappa|\xi|^{2} \tau} \hat{D}_{3}(\xi, \tau) d \tau \\
& \leq C\left|\left(\hat{v}_{0}\right)_{x_{1}}(\cdot, \xi)\right|_{L^{2}}^{2}+\left.C \int_{0}^{t}(1+\tau)^{\gamma} e^{\kappa|\xi|}\right|^{2} \tau\left(|\hat{v}(\cdot, \xi, \tau)|_{H^{1}}^{2}+|\xi|^{2}|\hat{v}(\cdot, \xi, \tau)|_{L^{2}}^{2}\right) d \tau,
\end{aligned}
$$

where $\gamma \geq 0$, and $C$ is a positive constant. These will be used in the next subsection. 
Proof of Corollary 3.2. By virtue of the Plancherel theorem, we have (2.3). Substituting (3.4) into (2.3), we obtain

$$
\begin{aligned}
\left\|\partial_{x^{\prime}}^{k} z(t)\right\|_{\mathcal{L}_{\alpha}^{2}}^{2} & \leq C \int_{\mathbb{R}_{\xi}^{n-1}}|\xi|^{2 k} e^{-\kappa|\xi|^{2} t}\left|\hat{z}_{0}(\cdot, \xi)\right|_{L_{\alpha}^{2}}^{2} d \xi \\
& \leq C \sup _{\xi \in \mathbb{R}_{\xi}^{n-1}}\left|\hat{z}_{0}(\cdot, \xi)\right|_{L_{\alpha}^{2}}^{2} \int_{\mathbb{R}_{\xi}^{n-1}}|\xi|^{2 k} e^{-\kappa|\xi|^{2} t} d \xi \\
& \leq C t^{-(n-1) / 2-k}\left\|z_{0}\right\|_{L_{\alpha}^{2}\left(L^{1}\right)^{\prime}}^{2}
\end{aligned}
$$

where $C$ is a positive constant. Here we used (2.2) and the simple inequality

$$
\int_{\mathbb{R}_{\xi}^{n-1}}|\xi|^{2 k} e^{-\kappa|\xi|^{2} t} d \xi \leq C t^{-(n-1) / 2-k}
$$

with a constant $C$. By applying the same argument to the pointwise estimates (3.5) and (3.6) we can derive the decay estimate (3.8) and (3.9), respectively. Thus this completes the proof.

\subsection{Weighted energy method}

In the last subsection, we restrict to the non-degenerate case $f_{1}^{\prime}(0)<0$ and apply the weighted energy method to the problems (3.2) and (3.3). This yields sharp pointwise estimates of solutions to (3.2). We use the weighted space $L_{\alpha}^{2}\left(\mathbb{R}_{+}\right)(\alpha \geq 0)$ for $x_{1} \in \mathbb{R}_{+}$in the normal direction and this gives the additional decay $(1+t)^{-\alpha / 2}$. The result is stated as follows.

Theorem 3.3 (Pointwise estimate). Let $f_{1}^{\prime}(0)<0$ and let $\phi\left(x_{1}\right)$ be a stationary solution with $\phi_{x_{1}}>0$. Let $\alpha \geq 0$ and suppose that $\hat{z}_{0}(\cdot, \xi) \in L_{\alpha}^{2}\left(\mathbb{R}_{+}\right)$and $\left(\hat{z}_{0}\right)_{x_{1}}(\cdot, \xi) \in H^{1}\left(\mathbb{R}_{+}\right)$for each $\xi \in \mathbb{R}_{\xi}^{n-1}$. Then the solution to the problem (3.2) verifies the pointwise estimate

$$
\begin{gathered}
|\hat{z}(\cdot, \xi, t)|_{L^{2}} \leq\left. C(1+t)^{-\alpha / 2} e^{-\kappa \mid \xi}\right|^{2} t\left|\hat{z}_{0}(\cdot, \xi)\right|_{L_{\alpha^{\prime}}^{2}} \\
\left|\hat{z}_{x_{1}}(\cdot, \xi, t)\right|_{L^{2}} \leq\left. C(1+t)^{-\alpha / 2} e^{-\kappa|\xi|}\right|^{2} t\left(\left|\hat{z}_{0}(\cdot, \xi)\right|_{L_{\alpha}^{2}}+\left|\left(\hat{z}_{0}\right)_{x_{1}}(\cdot, \xi)\right|_{L^{2}}\right), \\
\left|\hat{z}_{x_{1} x_{1}}(\cdot, \xi, t)\right|_{L^{2}} \leq C(1+t)^{-\alpha / 2} e^{-\kappa|\xi|^{2} t}\left(\left|\hat{z}_{0}(\cdot, \xi)\right|_{L_{\alpha}^{2}}+\left|\left(\hat{z}_{0}\right)_{x_{1}}(\cdot, \xi)\right|_{H^{1}}\right)
\end{gathered}
$$

for $\xi \in \mathbb{R}_{\xi}^{n-1}$ and $t \geq 0$, where the norms $|\cdot|_{L^{2}},|\cdot|_{H^{1}}$ and $|\cdot|_{L_{\alpha}^{2}}$ are with respect to $x_{1} \in \mathbb{R}_{+}$, and $C$ and $\kappa$ are positive constants.

As an easy consequence, we have the following decay estimate.

Corollary 3.4 (Decay estimate). Assume the same conditions of Theorem 3.3. Let $z_{0} \in L_{\alpha}^{2}\left(L^{1}\right)$ and $\left(z_{0}\right)_{x_{1}} \in H^{1}\left(L^{1}\right)$ for $\alpha \geq 0$. Then the solution to the problem (3.1), (2.6), (2.7) satisfies the decay estimate

$$
\begin{aligned}
& \left\|\partial_{x^{\prime}}^{k} z(t)\right\|_{\mathcal{L}^{2}} \leq C(1+t)^{-\alpha / 2} t^{-(n-1) / 4-k / 2}\left\|z_{0}\right\|_{L_{\alpha}^{2}\left(L^{1}\right)}, \\
& \left\|\partial_{x^{\prime}}^{k} z_{x_{1}}(t)\right\|_{\mathcal{L}^{2}} \leq C(1+t)^{-\alpha / 2} t^{-(n-1) / 4-k / 2}\left(\left\|z_{0}\right\|_{L_{\alpha}^{2}\left(L^{1}\right)}+\left\|\left(z_{0}\right)_{x_{1}}\right\|_{L^{2}\left(L^{1}\right)}\right), \\
& \left\|\partial_{x^{\prime}}^{k} z_{x_{1} x_{1}}(t)\right\|_{\mathcal{L}^{2}} \leq C(1+t)^{-\alpha / 2} t^{-(n-1) / 4-k / 2}\left(\left\|z_{0}\right\|_{L_{\alpha}^{2}\left(L^{1}\right)}+\left\|\left(z_{0}\right)_{x_{1}}\right\|_{H^{1}\left(L^{1}\right)}\right)
\end{aligned}
$$


for $t>0$, where $k \geq 0$ is an integer and $C$ is a positive constant.

The proof of Corollary 3.4 is completely same as the proof of Corollary 3.2 and omitted here.

Proof of Theorem 3.3. We use the weighted energy method to the problems (3.2) and (3.3) formulated in the partial Fourier space. Our computation is similar to the one used in $[4,10$, $12,14]$ and divide into four steps.

Step 1. First, we show the following space-time weighted energy inequality:

$$
\begin{aligned}
& (1+t)^{\gamma} e^{\kappa|\xi|^{2} t}|\hat{z}(\cdot, \xi, t)|_{L_{\beta}^{2}}^{2}+\left.\int_{0}^{t}(1+\tau)^{\gamma} e^{\kappa \mid \xi}\right|^{2} \tau\left(\hat{\mathbf{D}}_{\beta}(\xi, \tau)+\beta|\hat{z}(\cdot, \xi, \tau)|_{L_{\beta-1}^{2}}^{2}\right) d \tau \\
& \leq C\left|\hat{z}_{0}(\cdot, \xi)\right|_{L_{\beta}^{2}}^{2}+\left.\gamma C \int_{0}^{t}(1+\tau)^{\gamma-1} e^{\kappa \mid \xi}\right|^{2} \tau|\hat{z}(\cdot, \xi, \tau)|_{L_{\beta}^{2}}^{2} d \tau
\end{aligned}
$$

for $\gamma \geq 0$ and $0 \leq \beta \leq \alpha$, where

$$
\hat{\mathbf{D}}_{\beta}=\left|\hat{z}_{x_{1}}\right|_{L_{\beta}^{2}}^{2}+|\xi|^{2}|\hat{z}|_{L_{\beta}^{2}}^{2}+\left|\sqrt{\phi_{x_{1}}} \hat{z}\right|_{L_{\beta}^{2}}^{2}
$$

and $C$ and $\kappa$ are positive constants. Notice that $\hat{\mathbf{D}}_{0}$ coincides with $\hat{D}_{1}$ in (3.14).

To prove (3.31), we use the equality (3.11). Notice that, by virtue of $(2.9)_{1}$ in Lemma 2.2, we have

$$
-\mathcal{F}_{1} \geq c_{2}|\hat{z}|^{2}-C\left|\hat{z}_{x_{1}}\right|^{2}
$$

with positive constants $c_{2}$ and $C$. Now we multiply (3.11) by $\left(1+x_{1}\right)^{\beta}(0 \leq \beta \leq \alpha)$ to get

$$
\begin{aligned}
\frac{1}{2} \frac{\partial}{\partial t}\{(1 & \left.\left.+x_{1}\right)^{\beta}|\hat{z}|^{2}\right\}+\left\{\left(1+x_{1}\right)^{\beta} \mathcal{F}_{1}\right\}_{x_{1}} \\
& +\left(1+x_{1}\right)^{\beta} \mathcal{D}_{1}+\beta\left(1+x_{1}\right)^{\beta-1}\left(-\mathcal{F}_{1}\right)=0 .
\end{aligned}
$$

We integrate this equality over $x_{1} \in \mathbb{R}_{+}$and use (3.12) and (3.32), obtaining

$$
\frac{\partial}{\partial t}|\hat{z}|_{L_{\beta, w}^{2}}^{2}+c_{1} \hat{\mathbf{D}}_{\beta}+2 \beta c_{2}|\hat{z}|_{L_{\beta-1}^{2}}^{2}-\left(w f_{1}\right)^{\prime}\left(u_{b}\right)|\hat{z}(0, \xi, t)|^{2} \leq \beta C\left|\hat{z}_{x_{1}}\right|_{L_{\beta-1}^{2}}^{2}
$$

for $0 \leq \beta \leq \alpha$, where we define

$$
|v|_{L_{\beta, w}^{2}}=\left(\int_{0}^{\infty}\left(1+x_{1}\right)^{\beta} w\left(\phi\left(x_{1}\right)\right)\left|v\left(x_{1}\right)\right|^{2} d x_{1}\right)^{1 / 2},
$$

and $C$ is a positive constant. Here, by virtue of $(2.9)_{1}$, the last term of the left-hand side of (3.33) is positive. We now observe that

$$
|a|_{L_{\beta-1}^{2}}^{2} \leq \epsilon|a|_{L_{\beta}^{2}}^{2}+C_{\epsilon}|a|_{L^{2}}^{2}
$$

for any $\epsilon>0$, where $C_{\epsilon}$ is a constant depending on $\epsilon$. We apply this inequality to the term on the right-hand side of (3.33) by taking $a=\hat{z}_{x_{1}}$. Noting that $\hat{\mathbf{D}}_{\beta} \geq\left|\hat{z}_{x_{1}}\right|_{L_{\beta}^{2}}^{2}$, we choose $\epsilon>0$ so small that $\alpha C \epsilon \leq c_{1}$. This yields

$$
\frac{\partial}{\partial t}|\hat{z}|_{L_{\beta, w}^{2}}^{2}+c_{3} \hat{\mathbf{D}}_{\beta}+2 \beta c_{2}|\hat{z}|_{L_{\beta-1}^{2}}^{2} \leq \beta C\left|\hat{z}_{x_{1}}\right|_{L^{2}}^{2}
$$


for constants $c_{3}$ and C. Multiplying (3.34) by $\left.e^{\kappa \mid \xi}\right|^{2} t(\kappa>0)$, we obtain

$$
\frac{\partial}{\partial t}\left\{e^{\left.\kappa|\xi|\right|^{2} t}|\hat{z}|_{L_{\beta, w}^{2}}^{2}\right\}+e^{\kappa|\xi|^{2} t}\left(c_{3} \hat{\mathbf{D}}_{\beta}-\kappa|\xi|^{2}|\hat{z}|_{L_{\beta}^{2}}^{2}\right)+2 \beta c_{2} e^{\kappa|\xi|^{2} t}|\hat{z}|_{L_{\beta-1}^{2}}^{2} \leq \beta C e^{\kappa|\xi|^{2} t}\left|\hat{z}_{x_{1}}\right|_{L^{2}}^{2} .
$$

As in (3.15), we choose $\kappa>0$ such that $\kappa \leq c_{3}$. Then we multiply the resulting inequality by $(1+t)^{\gamma}(\gamma \geq 0)$ and integrate over $[0, t]$. This yields

$$
\begin{gathered}
\left.(1+t)^{\gamma} e^{\kappa \mid \xi}\right|^{2} t|\hat{z}(\cdot, \xi, t)|_{L_{\beta}^{2}}^{2}+\left.\int_{0}^{t}(1+\tau)^{\gamma} e^{\kappa \mid \xi}\right|^{2} \tau\left(\hat{\mathbf{D}}_{\beta}(\xi, \tau)+\beta|\hat{z}(\cdot, \xi, \tau)|_{L_{\beta-1}^{2}}^{2}\right) d \tau \\
\leq C\left|\hat{z}_{0}(\cdot, \xi)\right|_{L_{\beta}^{2}}^{2}+\left.\gamma C \int_{0}^{t}(1+\tau)^{\gamma-1} e^{\kappa \mid \xi}\right|^{2} \tau|\hat{z}(\cdot, \xi, \tau)|_{L_{\beta}^{2}}^{2} d \tau \\
+\left.\beta C \int_{0}^{t}(1+\tau)^{\gamma} e^{\kappa \mid \xi}\right|^{2} \tau\left|\hat{z}_{x_{1}}(\cdot, \xi, \tau)\right|_{L^{2}}^{2} d \tau,
\end{gathered}
$$

where $C$ is a positive constant. Here the last term on the right-hand side of (3.35) is already estimated in (3.25) because $\left|\hat{z}_{x_{1}}\right|_{L^{2}}^{2} \leq \hat{\mathbf{D}}_{0}=\hat{D}_{1}$. Therefore the proof of (3.31) is complete.

Step 2. Next we show the following estimate for $\alpha \geq 0$ :

$$
\begin{aligned}
& \left.(1+t)^{l} e^{\kappa \mid \xi}\right|^{2} t|\hat{z}(\cdot, \xi, t)|_{L_{\alpha-l}^{2}}^{2} \\
& +\int_{0}^{t}(1+\tau)^{l} e^{\kappa|\xi|^{2} \tau}\left(\hat{\mathbf{D}}_{\alpha-l}(\xi, \tau)+(\alpha-l)|\hat{z}(\cdot, \xi, \tau)|_{L_{\alpha-l-1}^{2}}^{2}\right) d \tau \leq C\left|\hat{z}_{0}(\cdot, \xi)\right|_{L_{\alpha}^{2}}^{2}
\end{aligned}
$$

for each integer $l$ with $0 \leq l \leq[\alpha]$, where $C$ and $\kappa$ are positive constants. Note that if $\alpha \geq 0$ is an integer, then (3.36) with $l=\alpha$ gives the desired estimate (3.28).

We prove (3.36) by induction with respect to the integer $l$ with $0 \leq l \leq[\alpha]$. First we put $\gamma=0$ and $\beta=\alpha$ in (3.31). This shows that (3.36) holds true for $l=0$. Now, let $1 \leq j \leq[\alpha]$ (for $\alpha \geq 1$ ) and suppose that (3.36) holds true for $l=j-1$. In particular, we suppose that

$$
\left.\int_{0}^{t}(1+\tau)^{j-1} e^{\kappa \mid \xi}\right|^{2} \tau|\hat{z}(\cdot \xi \xi, \tau)|_{L_{\alpha-j}^{2}}^{2} d \tau \leq C\left|\hat{z}_{0}(\cdot, \xi)\right|_{L_{\alpha}^{2}}^{2} .
$$

Then we prove (3.36) for $l=j$. To this end, we put $\gamma=j$ and $\beta=\alpha-j$ in (3.31). This gives

$$
\begin{aligned}
& (1+t)^{j} e^{\kappa|\xi|^{2} t}|\hat{z}(\cdot, \xi, t)|_{L_{\alpha-j}^{2}}^{2} \\
& +\int_{0}^{t}(1+\tau)^{j} e^{\kappa|\xi|^{2} \tau}\left(\hat{\mathbf{D}}_{\alpha-j}(\xi, \tau)+(\alpha-j)|\hat{z}(\cdot, \xi, \tau)|_{L_{\alpha-j-1}^{2}}^{2}\right) d \tau \\
& \leq C\left|\hat{z}_{0}(\cdot, \xi)\right|_{L_{\alpha-j}^{2}}^{2}+\left.j C \int_{0}^{t}(1+\tau)^{j-1} e^{\kappa \mid \xi}\right|^{2} \tau|\hat{z}(\cdot, \xi, \tau)|_{L_{\alpha-j}^{2}}^{2} d \tau \leq C\left|\hat{z}_{0}(\cdot, \xi)\right|_{L_{\alpha}^{2}}^{2}
\end{aligned}
$$

where we used (3.37) in the last estimate. This shows that (3.36) holds true also for $l=j$ and therefore the proof of (3.36) is complete.

Step 3. Next, when $\alpha>0$ is not an integer, we show that

$$
\begin{aligned}
& (1+t)^{\gamma} e^{\kappa|\xi|^{2} t}|\hat{z}(\cdot, \xi, t)|_{L^{2}}^{2}+\int_{0}^{t}(1+\tau)^{\gamma} e^{\kappa|\xi|^{2} \tau} \hat{D}_{1}(\xi, \tau) d \tau \\
& \leq C(1+t)^{\gamma-\alpha}\left|\hat{z}_{0}(\cdot, \xi)\right|_{L_{\alpha}^{2}}^{2}
\end{aligned}
$$


for $\gamma>\alpha$, where $\hat{D}_{1}$ is defined in (3.12), and $C$ and $\kappa$ are positive constants. Notice that (3.38) gives the desired estimate (3.28) even if $\alpha>0$ is not an integer.

To prove (3.38), we recall the inequality (3.25) which is the same as (3.31) with $\beta=0$. We need to estimate the second term on the right-hand side of (3.25). This can be done by applying the technique due to Nishikawa in [10]. When $\alpha>0$ is not an integer, we have from (3.36) with $l=[\alpha]$ that

$$
\begin{aligned}
(1+t)^{[\alpha]} e^{\left.\kappa|\xi|\right|^{2} t}|\hat{z}(\cdot, \xi, t)|_{L_{\alpha-[\alpha]}^{2}}^{2} & \leq C\left|\hat{z}_{0}(\cdot, \xi)\right|_{L_{\alpha}^{2},}^{2} \\
\int_{0}^{t}(1+\tau)^{[\alpha]} e^{\kappa|\xi|^{2} \tau}|\hat{z}(\cdot, \xi, \tau)|_{L_{\alpha-[\alpha]-1}^{2}}^{2} & \leq C\left|\hat{z}_{0}(\cdot, \xi)\right|_{L_{\alpha}^{2},}^{2,}
\end{aligned}
$$

where $C$ is a positive constant. Now, using a simple interpolation inequality $|a|_{L^{2}} \leq$ $|a|_{L_{\theta-1}^{2}}^{\theta}|a|_{L_{\theta}^{2}}^{1-\theta}(0 \leq \theta \leq 1)$ and the Hölder inequality, we see that

$$
\begin{aligned}
& \int_{0}^{t}(1+\tau)^{\lambda}|a(\tau)|_{L^{2}}^{2} d \tau \\
& \leq\left(\int_{0}^{t}(1+\tau)^{\mu}|a(\tau)|_{L_{\theta-1}^{2}}^{2} d \tau\right)^{\theta}\left(\int_{0}^{t}(1+\tau)^{v}|a(\tau)|_{L_{\theta}^{2}}^{2} d \tau\right)^{1-\theta},
\end{aligned}
$$

provided that $\lambda=\mu \theta+v(1-\theta)$ with $0 \leq \theta \leq 1$. We use (3.40) for $a=\left.e^{\kappa \mid \xi}\right|^{2} t / 2 \hat{z}\left(x_{1}, \xi, t\right)$, $\theta=\alpha-[\alpha], \lambda=\gamma-1, \mu=[\alpha]$ and the corresponding $v$ determined by $\lambda=\mu \theta+\nu(1-\theta)$. Then, using (3.39), we arrive at the estimate

$$
\begin{aligned}
& \left.\int_{0}^{t}(1+\tau)^{\gamma-1} e^{\kappa \mid \xi}\right|^{2} \tau|\hat{z}(\cdot, \xi, \tau)|_{L^{2}}^{2} d \tau \\
& \leq C\left|\hat{z}_{0}(\cdot, \xi)\right|_{L_{\alpha}^{2}}^{2}\left(\int_{0}^{t}(1+\tau)^{\nu-[\alpha]} d \tau\right)^{1-\theta} \leq C(1+t)^{\gamma-\alpha}\left|\hat{z}_{0}(\cdot, \xi)\right|_{L_{\alpha}^{2},}^{2}
\end{aligned}
$$

where we have used the fact that $(v-[\alpha]+1)(1-\theta)=\gamma-\alpha$. Substituting this estimate into (3.25), we get the desired estimate (3.38).

Step 4. Finally, we prove (3.29) and (3.30). Employing (3.38), we can estimate the last term of the right-hand side of (3.26). Namely, we obtain

$$
\begin{aligned}
& (1+t)^{\gamma} e^{\kappa|\xi|^{2} t}|\hat{v}(\cdot, \xi, t)|_{L^{2}}^{2}+\int_{0}^{t}(1+\tau)^{\gamma} e^{\kappa|\xi|^{2} \tau} \hat{D}_{2}(\xi, \tau) d \tau \\
& \leq C\left|\hat{v}_{0}(\cdot, \xi)\right|_{L^{2}}^{2}+C(1+t)^{\gamma-\alpha}\left|\hat{z}_{0}(\cdot, \xi)\right|_{L_{\alpha}^{2}}^{2} \leq C(1+t)^{\gamma-\alpha}\left(\left|\hat{z}_{0}(\cdot, \xi)\right|_{L_{\alpha}^{2}}^{2}+\left|\hat{v}_{0}(\cdot, \xi)\right|_{L^{2}}^{2}\right)
\end{aligned}
$$

for $\gamma>\alpha$. Thus this yields (3.29).

On the other hand, by applying (3.38) and (3.41) to (3.27), we get

$$
\begin{gathered}
(1+t)^{\gamma} e^{\kappa|\xi|^{2} t}\left|\hat{v}_{x_{1}}(\cdot, \xi, t)\right|_{L^{2}}^{2}+\int_{0}^{t}(1+\tau)^{\gamma} e^{\kappa|\xi|^{2} \tau} \hat{D}_{3}(\xi, \tau) d \tau \\
\leq C(1+t)^{\gamma-\alpha}\left(\left|\hat{z}_{0}(\cdot, \xi)\right|_{L_{\alpha}^{2}}^{2}+\left|\hat{v}_{0}(\cdot, \xi)\right|_{H^{1}}^{2}\right)
\end{gathered}
$$

for $\gamma>\alpha$. This means that (3.30). Hence this completes the proof of Theorem 3.3. 


\section{References}

[1] Hashimoto, I. \& Matsumura, A. (2007). Large time behavior of solutions to an initial boundary value problem on the half line for scalar viscous conservation law, Methods Appl. Anal., Vol. 14, 45-60.

[2] Hashimoto, I. \& Ueda, Y. (2011). Anti-derivative method in the half space and application to damped wave equations with non-convex convection, to appear in Kyushu Journal of Mathematics.

[3] Il'in, A. M. \& Oleinik, O. A. (1964). Behavior of the solution of the Cauchy problem for certain quasilinear equations for unbounded increase of the time, Amer. Math. Soc. Transl., Vol. 42, 19-23.

[4] Kawashima, S. \& Matsumura, A. (1985). Asymptotic stability of traveling wave solutions of systems for one-dimensional gas motion, Commun. Math. Phys., Vol. 101, 97-127.

[5] Kawashima, S.; Nishibata, S. \& Nishikawa, M. (2003). Asymptotic stability of stationary waves for two-dimensional viscous conservation laws in half plane, Discrete Contin. Dyn. Syst., Suppl., 469-476.

[6] Kawashima, S.; Nishibata, S. \& Nishikawa, M. (2004). $L^{p}$ energy method for multi-dimensional viscous conservation laws and application to the stability of planar waves, J. Hyperbolic Differential Equations, Vol. 1, 581-603.

[7] Kawashima, S.; Nishibata, S. \& Nishikawa, M. (2004). Asymptotic stability of stationary waves for multi-dimensional viscous conservation laws in half space, preprint.

[8] Liu T.-P.; Matsumura, A. \& Nishihara, K. (1998). Behaviors of solutions for the Burgers equation with boundary corresponding to rarefaction waves, SIAM J. Math. Anal., Vol. 29, 293-308.

[9] Liu, T.-P. \& Nishihara, K. (1997). Asymptotic behavior for scalar viscous conservation laws with boundary effect, J. Differential Equations, Vol. 133, 296-320.

[10] Nishikawa, M. (1998). Convergence rate to the traveling wave for viscous conservation laws, Funkcial. Ekvac., Vol. 41, 107-132.

[11] Ueda, Y. (2008). Asymptotic stability of stationary waves for damped wave equations with a nonlinear convection term, Adv. Math. Sci. Appl., Vol. 18, No. 1, 329-343.

[12] Ueda, Y.; Nakamura, T. \& Kawashima, S. (2008). Stability of planar waves for damped wave equations with nonlinear convection in multi-dimensional half space, Kinetic and Related Models, Vol. 1, 49-64.

[13] Ueda, Y.; Nakamura, T. \& Kawashima, S. (2010). Stability of degenerate stationary waves for viscous gases, Arch. Rational Mech. Anal., Vol. 198, 735-762.

[14] Ueda, Y.; Nakamura, T. \& Kawashima, S. (2011). Energy method in the partial Fourier space and application to stability problems in the half space, J. Differential Equations, Vol. 250, 1169-1199. 


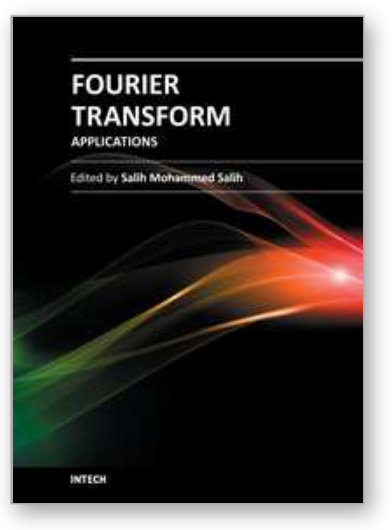

\author{
Fourier Transform Applications \\ Edited by Dr Salih Salih
}

ISBN 978-953-51-0518-3

Hard cover, 300 pages

Publisher InTech

Published online 25, April, 2012

Published in print edition April, 2012

The book focuses on Fourier transform applications in electromagnetic field and microwave, medical applications, error control coding, methods for option pricing, and Helbert transform application. It is hoped that this book will provide the background, reference and incentive to encourage further research and results in these fields as well as provide tools for practical applications. It provides an applications-oriented analysis written primarily for electrical engineers, control engineers, signal processing engineers, medical researchers, and the academic researchers. In addition the graduate students will also find it useful as a reference for their research activities.

\title{
How to reference
}

In order to correctly reference this scholarly work, feel free to copy and paste the following:

Yoshihiro Ueda (2012). Application of the Weighted Energy Method in the Partial Fourier Space to Linearized Viscous Conservation Laws with Non-Convex Condition, Fourier Transform Applications, Dr Salih Salih (Ed.), ISBN: 978-953-51-0518-3, InTech, Available from: http://www.intechopen.com/books/fourier-transformapplications/application-of-the-energy-method-in-the-partial-fourier-space-to-viscous-conservation-laws-withnon

\section{INTECH}

open science | open minds

\section{InTech Europe}

University Campus STeP Ri

Slavka Krautzeka 83/A

51000 Rijeka, Croatia

Phone: +385 (51) 770447

Fax: +385 (51) 686166

www.intechopen.com

\section{InTech China}

Unit 405, Office Block, Hotel Equatorial Shanghai

No.65, Yan An Road (West), Shanghai, 200040, China

中国上海市延安西路65号上海国际贵都大饭店办公楼405单元

Phone: +86-21-62489820

Fax: $+86-21-62489821$ 
(C) 2012 The Author(s). Licensee IntechOpen. This is an open access article distributed under the terms of the Creative Commons Attribution 3.0 License, which permits unrestricted use, distribution, and reproduction in any medium, provided the original work is properly cited. 